\title{
Formação em Odontologia: desafios para o desenvolvimento docente e efetiva inclusão do Sistema Único de Saúde
}

\author{
Talitha Rodrigues Ribeiro Fernandes Pessoa*; Luiz Roberto Augusto Noro**
}

* Doutora, Professora Adjunta, Departamento de Clínica e Odontologia Social, UFPB

** Pós-doutorado em Educação, Professor do Departamento de Odontologia e do Programa de Pós-graduação em Saúde Coletiva, UFRN

Recebido em 12/03/2019. Aprovado em 05/01/2020.

\begin{abstract}
RESUMO
As dificuldades enfrentadas para a efetivação das Diretrizes Curriculares Nacionais dos cursos de graduação de Odontologia são reflexos da dificuldade de operar mudanças num sistema educacional que permaneceu por décadas refém do currículo mínimo e com resistência ideológica em aderir a propostas que se baseiem na produção do cuidado. Visando enfrentar essas dificuldades, o presente estudo buscou verificar a aplicabilidade de critérios de avaliação da formação em Odontologia com foco na formação voltada para o Sistema Único de Saúde (SUS) e na abordagem pedagógica. Realizou-se estudo documental dos Projetos Pedagógicos dos Cursos, aplicação de matriz de critérios e entrevista com os coordenadores de quatro cursos de Odontologia de instituições públicas e privadas. A percepção dos coordenadores configurou-se espaço privilegiado para reflexão sobre os avanços e limitações do projeto pedagógico, servindo como processo de autoavaliação sobre os principais desafios a serem enfrentados. É nítida a necessidade de nova regulamentação prevendo a obrigatoriedade de vínculo formal com o SUS, em especial no estágio curricular, assim como estratégias que contribuam efetivamente para o desenvolvimento docente permanente, viabilizando a construção de currículos integrados e incorporação efetiva de metodologias ativas de aprendizagem. Descritores: Educação em Odontologia. Currículo. Aprendizagem. Sistema Único de Saúde.
\end{abstract}

\section{INTRODUÇÃO}

As dificuldades enfrentadas para a efetivação das Diretrizes Curriculares Nacionais (DCN) dos cursos de graduação de Odontologia são reflexos tanto da complexidade de operar mudanças num sistema educacional que permaneceu por décadas refém das definições do currículo mínimo, como da resistência ideológica em aderir a propostas que se baseiem na produção do cuidado na acepção mais ampla do conceito de saúde, em contraposição à 
racionalidade hegemônica calcada na doença e na produção de procedimentos ${ }^{1}$.

Papel estratégico poderiam desempenhar os programas de pós-graduação, se não com a abertura de linhas de pesquisa em educação, ao menos revendo o lugar e a dimensão que a discussão sobre formação tem ocupado em seus currículos, uma vez que há quase uma negligência de questões que apontam a relevância da formação pedagógica nesse âmbito ${ }^{2}$. Não se pode esperar uma transformação espontânea das instituições acadêmicas na direção assinalada pelas DCN para estimular as mudanças na formação profissional em saúde de acordo com interesses e necessidades da população, contraditório ao modelo hegemônico da formação em Odontologia ainda hoje ${ }^{3}$.

Para se atingir essa melhoria da qualidade da formação é fundamental a adoção de mecanismos de avaliação que possam induzir a incorporação dos pressupostos das DCN pelos cursos de Odontologia. De forma ampla, esta estratégia tem sido definida ao longo dos tempos por meio do Sistema Nacional de Avaliação da Educação Superior (SINAES), o qual tem como figura fundamental a autoavaliação $0^{4,5}$.

Somente um processo autoavaliativo participativo, que acompanhe a implementação das ações, pode adequá-las a fim de alcançar efetividade e impacto com as mudanças requeridas na formação de um profissional diferenciado para atender as necessidades de saúde da população brasileira $^{6}$.

Buscando sinalizar alternativas que extrapolem as avaliações oficiais, permitindo que as próprias instituições de educação superior assumam papel de protagonismo na sua proposta educativa, o objetivo do presente estudo foi verificar a validade e aplicabilidade de critérios de autoavaliação da formação em Odontologia com base nos princípios das Diretrizes Curriculares Nacionais, em especial na formação voltada para o Sistema Único de Saúde e à abordagem pedagógica.

\section{METODOLOGIA}

O presente estudo foi realizado em três fases interdependentes, das quais participaram quatro coordenadores de cursos de Odontologia, sendo dois de instituições públicas e dois de instituições privadas.

A primeira fase consistiu no preenchimento de matriz de critérios previamente validada, com aspectos relativos à formação generalista, orientação do cuidado, integração ensino-serviço e abordagem pedagógica ${ }^{7}$.

A segunda fase consistiu na análise do projeto pedagógico dos cursos participantes, a qual permitiu a compreensão do contexto, da organização curricular e da proposta pedagógica.

$\mathrm{Na}$ terceira fase foram realizadas entrevistas com coordenadores, as quais foram avaliadas por meio da análise de conteúdo ${ }^{8}$, tendo como referencial roteiro de entrevista semiestruturada, com perguntas e aspectos a serem detalhados para cada uma das subdimensões avaliadas na primeira fase do estudo. Estrategicamente, para esta etapa foram aprofundadas as dimensões "integração ensino-serviço" e "abordagem pedagógica", considerando serem as mais desafiadoras na efetivação das DCN.

Quanto aos princípios éticos de pesquisa em seres humanos, o projeto seguiu as orientações da Resolução 466/2012 do Conselho Nacional de Saúde, tendo sido aprovado pelo parecer 292.805 e Certificado de Apresentação para Apreciação Ética (CAAE):13989213.0.0000.5292. do Comitê de Ética em Pesquisa do Hospital Onofre Lopes.

\section{RESULTADOS E DISCUSSÃO}

A análise da matriz de critérios das dimensões permitiu uma primeira aproximação das condições dos cursos de Odontologia analisados, servindo como referência para direcionamento da pesquisa nas duas fases subsequentes. 
A avaliação dos Projetos Pedagógicos de Curso (PPC) representou um grande desafio, uma vez que todos apresentaram uma estrutura bastante semelhante. Todos os PPC avaliados apontaram expressamente as DCN como eixo condutor na sua construção e, principalmente nos aspectos relacionados ao perfil do egresso e às competências e habilidades gerais e específicas. Todos apresentaram o cumprimento de $20 \%$ de carga horária total para os estágios curriculares, entretanto, nos quatro cursos esta carga horária inclui as clínicas integradas do curso, sendo que em alguns deles representam a maior parte dos estágios. Embora esteja ressaltada nos PPC a importância da formação voltada para o SUS, como preconizam as DCN, a forma como ocorre a inserção dos estudantes e o desenvolvimento das atividades não é claramente explicitada nos projetos pedagógicos.

A possibilidade de encontrar nos PPC concordância com as DCN para além da redação destas no texto do projeto foi mais evidente quando da avaliação da estrutura curricular. Um olhar mais atento a este componente do PPC permitiu a visualização das tentativas de integração e/ou flexibilização curricular pela observação da forma como os componentes são ofertados.

No que diz respeito à abordagem pedagógica, foi verificado frequentemente nos PPC a indicação para utilização de metodologias ativas, interativas e centradas no aluno como sujeito da aprendizagem, e do professor no papel de facilitador da aprendizagem com responsabilização pela condução técnico-pedagógica deste processo. Ainda, estratégias como educação pela pesquisa, experiências coletivas e multidisciplinares, diversificação dos cenários de aprendizagem são apontadas para esta inovação. Contudo, poucas especificações sobre como são efetivadas estas estratégias no cotidiano dos cursos foram encontradas, restringindo-se à citação de algumas metodologias, como a da problematização.
Em relação à avaliação do processo ensino aprendizagem foi constatado que a proposta de avaliação da aprendizagem não acompanha as inovações relacionadas à metodologias ativas, restringindo-as às normas institucionais vigentes enfatizando os instrumentos utilizados (escala de notas, periodicidade e quantidade de registros).

Foi possível identificar nos PPC apontamentos quanto à necessidade de enfrentar os desafios para a implementação de políticas de qualificação docente que vão além da formação stricto sensu e contemplem a atualização pedagógica.

$\mathrm{Na}$ terceira fase do estudo, aspectos sinalizados nos PPC e conceitos emitidos na matriz inicial puderam ser aprofundados, considerando as dificuldades de operacionalização do que está proposto no documento. Para melhor compreensão dessas análises, elas foram definidas em 2 categorias, representadas pelas dimensões "Integração Ensino-Serviço" e "Abordagem Pedagógica". Os coordenadores são identificados de $\mathrm{C} 1$ a C4.

\section{Categoria Integração Ensino-Serviço}

Com relação às atividades de estágios, a integração ensino serviço foi verificada pela constituição de convênios entre as IES e os serviços, além do planejamento e pactuação das atividades.

"Nós temos um convênio entre a universidade e o governo do estado e é o que regulamenta o nosso atendimento no CEO regional... e em relação à prefeitura, há um convênio entre a universidade e a prefeitura." $(\mathrm{C} 1)$

As atividades realizadas durante os estágios são bastante diversificadas e compreendem atividades coletivas e individuais desde promoção e prevenção de doenças nos primeiros semestres do curso até atendimento clínico realizado nos últimos anos. 
"Até o $6^{\circ}$ semestre, são conduzidos para dirigir atividades de caráter educativo, promoção de saúde. Mas todas as atividades que são de caráter clínico, que eu preciso da supervisão do professor de estágio, de dentista contratado no serviço de origem, o dentista da UBS ou o dentista da escola que vai fazer a supervisão, aí só no último ano." (C4)

Sem dúvida, os estágios são fundamentais para a formação em serviço, em especial ao considerar-se o SUS como cenário real de aprendizagem. A aproximação com a Atenção Primária tem permitido tanto a formação de profissionais que possam responder adequadamente às necessidades da população e de comprometimento social previsto pelo SUS quanto para a melhor compreensão do processo de cuidado 9 . Outras vantagens são a concretização do trabalho com autonomia, aproximando os estudantes de Odontologia da realidade dos serviços, dos demais profissionais da saúde e do cuidado com a comunidade ${ }^{10,11}$.

Dentro do critério "Vivências no SUS", vários cenários puderam ser identificados como campo de estágio, havendo limitações principalmente com relação à inserção dos estudantes no nível terciário de atenção, mas ressaltou-se que, na medida do possível, há uma integração entre os níveis vivenciados. Em um dos cursos avaliados todas as atividades clínicas ocorrem dentro do SUS.

"No nível primário e secundário sim, quase que por inteiro. $O$ terciário é um pouco mais complicado porque nós não tínhamos até então um serviço que fosse uma atenção terciária especifica." (C1)

Apesar dos claros avanços com relação à integração ensino-serviço, não foi consenso entre os coordenadores da pesquisa que os alunos conseguem compreender a complexidade do SUS em sua organização, princípios e diretrizes, muitas vezes trabalhados apenas na área da saúde coletiva. "Os aspectos relativos ao SUS são trabalhados teoricamente em sala de aula... e mesmo para nós que temos uma vivência muito menor nessa gestão em saúde pública." (C4)

Não é mais possível pensar na mudança na formação dos profissionais de saúde sem a discussão sobre a articulação ensino-serviçocomunidade. É fundamental compreender que na formação em saúde há um processo que deve permitir a abertura de caminhos, o qual, além de identificar as limitações, comprometa-se com a consolidação do SUS e com as demandas sociais ${ }^{12}$.

\section{Categoria Abordagem Pedagógica}

Verificou-se que quanto mais são trabalhadas e implementadas metodologias ativas e problematizadoras no curso, maior a tendência de se perceber mudanças na condução do processo de aprendizagem, sendo o contrário também verdadeiro.

"A gente hoje trabalha muito com as metodologias ativas, mas eu não saberia dizer se ela é a predominante. Porque a gente tem sim substituído esse modelo tradicional de ensino-aprendizagem." (C2) "A predominante ainda é a aula expositiva, discutidas na medida do possível." (C1)

Contudo, não foi verificada a tendência dos cursos em trabalhar com modelos pré-definidos de metodologias ativas, ficando sempre a critério do professor as definições metodológicas para reorientar a condução de acordo com sua iniciativa e criatividade.

“... então a gente fez a opção de trabalhar mais com a metodologia voltada para a problematização, porque dependeria muito mais da iniciativa, da criatividade, do envolvimento do professor do que uma determinação institucional." (C2)

Entretanto, mesmo em face às novas 
abordagens, observaram-se ainda incipientes alguns aspectos que fundamentam as metodologias ativas, como por exemplo, oportunizar e/ou estimular no aluno a autonomia e responsabilização sobre seu processo de aprendizagem.

"A gente tem isso ainda como uma coisa muito frágil, não sei se pelo perfil do aluno, ou pela relação aluno com professor, a gente vê assim que essa autonomia da busca pelo conhecimento do aluno não é uma coisa assim da forma como a gente queria." (C2)

Outro aspecto que chama atenção devido à resistência dos cursos às mudanças no que diz respeito à metodologia de ensino é a relação entre teoria e prática, na qual se verificou a predominância da tradicional dicotomia e fragmentação de saberes contraditórios à formação integrada e problematizadora que se propõe na atualidade. Nas atividades teóricas, a principal abordagem continua sendo a aula expositiva, na maioria das vezes com a condução de apenas um professor para um grande número de estudantes.

"Geralmente a teoria vem antes. A gente tenta integrar, ou às vezes antecipar a prática, ver quais são os problemas $e$ abordá-los na teoria, mas as nossas grades de horário na faculdade elas não permitem." (C1)

As metodologias ativas não podem ser confundidas meramente como uma nova tecnologia de ensino. Trata-se na realidade de compreender o processo de aprendizagem que permite protagonismo a todos os atores envolvidos, em especial alunos e professores. Para isso, a postura do professor deve privilegiar o exercício da liberdade de expressão e ação do aluno, a partir da reflexão crítica e da pesquisa, permitindo práxis que contemple a construção coletiva do aprendizado $^{13}$. Já o aluno estimula-se em buscar solução para impasses, promovendo seu próprio desenvolvimento científico e social ${ }^{14}$, produzindo tanto mudanças intrínsecas, quanto na sua relação com os usuários e a comunidade geral ${ }^{15}$.

Com relação à integração curricular preconizada pelas DCN, foi observado que há iniciativas de integração nas novas propostas curriculares, ainda muito limitada com o ciclo básico e com as ciências humanas, mas mais presente entre as especialidades odontológicas, principalmente por meio das clínicas de integração de complexidade crescente ao longo do curso.

"É uma integração muito frágil, porque a gente não tem integração das áreas básicas do curso... As outras áreas de ciências humanas elas informam o curso, mas elas não compõem o curso, essas disciplinas são pontuais onde eles estão, não tem nenhum tipo de projeto, uma articulação em conjunto. Eu vejo assim que a integração ela se deu entre as clínicas especializadas." (C2)

Nas atividades práticas, maior ênfase é dada aos espaços laboratoriais e de clínica, e se apresentou a tendência de manutenção da sequência tradicional na formação técnica em odontologia: aulas teóricas, atividades em laboratórios pré-clínicos e finalmente as clínicas.

"Nas disciplinas de saúde coletiva eu acho que tem a teoria concomitante com a prática com relação à visitação dos serviços. Nas outras, as disciplinas que são mais técnicas têm a teoria e os estudantes se destinam ao laboratório, ou laboratório de clínica." (C1)

Nas atividades clínicas do curso, relatou-se a priorização do planejamento qualificado de casos clínicos frente à execução de procedimentos, mesmo que esta última ainda seja uma prática para a formação clínica do estudante.

"Pelo menos tem a diretriz de que nenhum tratamento seja executado antes de um planejamento. Não o planejamento como uma sequência de atos operatórios, mas 
também com estudos de retomada da literatura para embasar a decisão." (C2)

Entretanto, foi constatado que, mesmo em clínicas integradas, a orientação docente se dá pela área de conhecimento do professor, o que se contrapõe à perspectiva de integralidade pressuposta na elaboração e execução de planejamentos clínicos. Nas atividades práticas, a relação é geralmente de um professor para cada dez alunos, mas esta pode ser prejudicada pelo fato de cada professor só orientar a sua especialidade nas clínicas integradas.

“... a gente não construiu ao longo desses anos de experiência essa integração efetiva. Então acaba que cada especialidade entra em um momento da formação. Muito pouco se conseguiu nessa articulação..." (C2)

A perspectiva da integralidade na formação do aluno é um dos maiores desafios considerando a formação do professor de Odontologia com base nas especialidades clínicas. Esta abordagem resulta num ensino restrito às técnicas de uma disciplina, também fortemente influenciada pela prática profissional clínica do professor, na maior parte das vezes referendada por sua atuação nesse tipo de mercado de trabalho ${ }^{16}$.

Com relação ao papel do professor, foi percebida a predominância de uma postura centralizadora deste, mesmo já sendo perceptíveis alguns avanços.

"Eu vejo que é um papel que está sendo construído. Nós temos um processo de transição daquele professor que a gente tem internalizado, aquele professor tradicional, detentor do saber, aquele de transmitir e nós estamos nos transformando nesse professor que é o mediador do conhecimento, que estimula o aluno, que desperta o interesse e que de uma certa forma trabalha com o potencial individual de cada aluno. (C2)
As maiores dificuldades na mudança de postura dos professores pareceram estar relacionadas à experiência pregressa destes ao vivenciar o ensino tradicional em sua formação, além da sensação de domínio e comodidade do ser professor utilizando metodologias tradicionais de ensino. Outra dificuldade apontada com relação ao papel do professor foram as múltiplas atribuições que tem que ser assumidas pelos docentes nas instituições.

"O professor tradicional é muito confortável nesse sentido, que é pouco questionado, a gente fica menos susceptível às questões que a gente não controla ... é dificil, quebrar o autoritarismo que a gente traz com a gente." (C2)

Portanto, constatou-se que o professor tem total autonomia para a condução dos processos de ensino em seus componentes curriculares frente às orientações legais e institucionais estabelecidas.

"Apesar de ter uma ementa, de ter um PPC, mas então a função do coordenador é verificar se aqueles conteúdos que estão nas ementas eles estejam presentes nas disciplinas." (C2)

Pôde-se ainda verificar a percepção de que o papel do professor na formação do profissional é também de cunho ético e humanístico.

"As nossas ações têm falado muito mais daquilo que está no papel. Eu acho que o aluno reflete um espelho do seu corpo docente e ele vai se tornar aquilo que o seu corpo docente transmite para ele também como homem, como ser humano." (C4)

Foram levantadas questões sobre a insuficiência da formação do cirurgião-dentista para o exercício da docência, mesmo os que concluíram a formação stricto sensu. Adicionalmente, também foi levantada dificuldades quanto à formação para a orientação clínica desses professores pesquisadores.

"Os professores começam a ser 
"professor", às vezes, muito jovens $e$ geralmente com formação em pesquisa. Então acho que os dentistas se formam e concluem mestrado, doutorado, não tem uma disciplina docente no curso de pósgraduação." (C1)

Sem dúvida, o professor de Odontologia com sua limitada formação pedagógica, ao se deparar com o desafio de proporcionar experiências adequadas e significativas capazes de levar o aluno a uma atitude investigativa de curiosidade perante o mundo ${ }^{17}$, prática à qual não foi exposto em seu processo formativo, grande parte das vezes opta em repetir a forma e os valores que lhe foram transmitidos em sua própria formação. Fica clara a necessidade radical de se ressignificar o papel do professor, uma vez que deverá se apropriar de conhecimentos pedagógicos da didática, das estratégias para responsável exercício da docência, das dimensões subjetivas dos estudantes na condição de sujeitos ativos de sua aprendizagem, do contexto educativo, dos objetivos, finalidades e valores educativos, além dos fundamentos filosóficos e históricos da Educação ${ }^{16}$. Compreendendo que o professor da área da saúde busca coerência com seu papel de educador, concorda-se com a percepção de Silva e Ferreira ${ }^{18}$ para quem "a estrada agora tem mais curvas e ficou mais longa, mas o objetivo talvez não seja mais apenas chegar ao destino, mas aproveitar a viagem".

O desenvolvimento docente, para além da carreira profissional, deve se integrar com a possibilidade de revoluções nos diferentes momentos da vida de um professor, associado às perspectivas de desenvolvimento humano ${ }^{19}$ e a uma formação formal do professor, que inclua o trabalho colaborativo, em contexto de trabalho para desafiar culturas profissionais marcadas pelo isolamento ${ }^{20}$.

Ainda na perspectiva da abordagem pedagógica, foi percebido como predominante o modelo tradicional de avaliação do desempenho dos estudantes no processo de aprendizagem, no qual são utilizadas as provas escritas e a atribuição de notas teóricas e práticas. Algumas iniciativas para a implementação de uma avaliação mais coerente com a proposta pedagógica preconizada nas DCN e no PPC do curso foram sinalizadas, mas ainda de forma bastante incipiente.

"Isso varia de acordo com as disciplinas. Em geral são provas mesmo, teóricas $e$ avaliações práticas. Nas clínicas é que a avaliação é feita de forma diária, os professores lançam conceitos e esses conceitos são levados em consideração para que se obtenha uma nota." (C3)

Ao se reduzir o processo avaliativo a um conjunto de instrumentos para medir conhecimento ou acúmulo de informação reduz-se drasticamente a perspectiva da avaliação como indutora de aprendizagem significativa. É, portanto, fundamental refletir sobre as reais características de um processo avaliativo que diga respeito a nossas práticas pedagógicas ${ }^{21}$ e não somente promovendo respostas ao processo institucional, permitindo coerência entre as conquistas possibilitadas pela aprendizagem colaborativa, identificando claramente o investimento de professor e aluno para se atingir o objetivo desejado. Para isso, a avaliação deve ser encarada como um processo diário, contínuo e integrador da ação educativa, que respeite o aluno no seu processo de aprendizagem e estágio de desenvolvimento ${ }^{22}$.

A flexibilização curricular é proporcionada principalmente pela oferta de componentes curriculares optativos ou pelo aproveitamento de carga horária de atividades complementares realizadas a critério dos estudantes. Pôde-se perceber que algumas vezes esses recursos estão fragilizados nos cursos e em outras, potencializado. "Atualmente qualquer atividade que o nosso aluno faça, são horas contadas, a gente não tem um limite, apesar de ele ter 
de fazer no mínimo 100 horas, não tem um teto. Então a gente computa todos os certificados que ele leva: projeto de pesquisa, projeto de extensão, ação comunitária, participação em organização de congresso, apresentação de trabalhos, tudo que ele leva é aproveitado e é jogado no sistema como uma atividade complementar." (C2)

Muitas barreiras puderam ser percebidas no exercício da flexibilização curricular, principalmente relacionadas à estrutura física do curso e à falta de horários disponíveis pelos estudantes para a realização de outras atividades, seja pela alta carga horária do curso ou por motivos e interesses pessoais.

"Hoje a gente quase não consegue ter essa flexibilização por conta das dificuldades que nós estamos com relação ao espaço físico. Como há dificuldade de horário, as grades estão muito apertadas, não tem muita flexibilidade para isso não." (C1)

A flexibilização curricular configura-se como um dos mais interessantes mecanismos de permitir que o aluno "faça seu próprio caminho", considerando seus interesses e expectativas, além de possibilitar o exercício da autonomia, tanto na forma de componentes didáticos optativos como em projetos de extensão, pesquisa e monitoria.

Finalmente, foi percebido nos resultados das entrevistas um nítido distanciamento entre a proposta pedagógica expressa no PPC e orientada pelas DCN e a realidade executada na formação profissional em cada curso, em cada área, em cada componente curricular.

"Muito daquilo que a gente coloca no papel, e a gente coloca no papel como a gente acredita que deve que ser, na hora da prática, eu acho que a personalidade do corpo docente influencia mais na ação do que a palavra que está no papel.” (C4)

$\mathrm{O}$ presente estudo não teve o objetivo de avaliar comparativamente os cursos participantes, principalmente considerando tratar-se de uma amostra por conveniência, o que inviabiliza análises quantitativas dessa natureza.

\section{CONCLUSÕES}

Foi possível identificar o potencial da triangulação dos procedimentos metodológicos utilizados ao conferir maior clareza nos aspectos observados no preenchimento preliminar da matriz, no aprofundamento da leitura documental dos elementos do projeto pedagógico e da possibilidade de identificar elementos com clareza parcial, a partir das entrevistas.

Além disso, configurou-se, na percepção dos coordenadores, espaço privilegiado para reflexão sobre os avanços e limitações do projeto pedagógico de seu curso, servindo como processo de autoavaliação sobre os principais desafios a serem enfrentados na busca pelo preconizado nas DCN dos cursos de Odontologia.

Os resultados obtidos possibilitaram identificar a importância do projeto pedagógico de um curso de Odontologia para o desenvolvimento e a condução de todas as estratégias de orientação da formação de cirurgiões-dentistas. Ao mesmo tempo, por se tratar de um documento utilizado não somente para o planejamento e estruturação, mas também como referência para a regulação dos cursos (autorização, reconhecimento e renovação de reconhecimento), os PPC de todos os cursos garantem a legalidade prevista nesses processos.

Ao se analisar a dimensão "Integração ensino-serviço" é nítida a necessidade de nova regulamentação que seja coerente com a legislação do estágio e com o preceito de considerar a articulação com o Sistema Único de Saúde. Para isso, é fundamental que essa inserção no SUS seja obrigatória para todos os cursos, com carga horária adequada e compromisso institucional que vincule a universidade aos serviços por meio de convênio, conforme já definido nas Diretrizes Curriculares 
Nacionais dos cursos de graduação em Medicina ${ }^{23}$. Além disso, formar no Sistema Único de Saúde deve extrapolar os componentes curriculares vinculados à Saúde Coletiva, considerando toda a dinâmica da assistência odontológica, além da promoção de saúde e prevenção de doenças.

$\mathrm{Na}$ análise da dimensão "Abordagem Pedagógica" foi possível verificar que ainda existem várias barreiras que dificultam princípios previstos a mais de 10 anos nas DCN dos cursos de Odontologia. Tais limitações têm como elemento principal a falta de formação pedagógica dos professores e gestores dos cursos, na maioria das vezes limitada a participação em disciplinas isoladas em sua formação na Pós-graduação. É fundamental que os cursos (em especial o Núcleo Docente Estruturante) organizem atividades que efetivamente contribuam com o desenvolvimento docente permanente, permitindo acesso a alternativas contemporâneas da formação em saúde, com a perspectiva essencial de trabalho colaborativo entre os professores, o que favorece o fortalecimento das relações interpessoais e a integração curricular, assim como a coerência com o contexto vivenciado pelos atores do curso, em especial na perspectiva de resolver os principais problemas da comunidade a ele adscrita.

A construção de currículos integrados, com forte contribuição do processo avaliativo na indução da qualidade do aprendizado, por meio da utilização de metodologias ativas de aprendizagem que apelem para a compreensão do papel cidadão a ser desempenhado por um profissional de saúde somente pode ser atingida a partir da articulação de todos esses saberes e práticas.

\section{ABSTRACT \\ Dentistry training: challenges for teacher development and effective inclusion in the Unified Health System}

The difficulties faced in implementing the National Curriculum Guidelines for undergraduate Dentistry courses are reflections of the difficulty of operating changes in an educational system that has remained hostage to the minimum curriculum for decades and with ideological resistance in adhering to proposals based on the production of care. To face these difficulties, the present study sought to verify the applicability of evaluation criteria for training in Dentistry with a focus on training focused on Brazil's Unified Health System and the pedagogical approach. A documentary study of the Pedagogical Projects of the Courses, application of a matrix of criteria, and an interview with the coordinators of four Dentistry courses from public and private institutions was carried out. The coordinators' perception became a privileged space for reflection on the advances and limitations of the pedagogical project, serving as a self-assessment process on the main challenges to be faced. There is a clear need for new regulations providing for the mandatory formal bond with SUS, especially in the curricular internship, as well as strategies that effectively contribute to the permanent teaching development, enabling the construction of integrated curricula and effective incorporation of active learning methodologies.

Descriptors: Education, Dental. Curriculum. Learning. Unified Health System.

\section{REFERÊNCIAS}

1. Casotti E, Ribeiro VMB, Gouvêa MV. Educação em odontologia no Brasil: produção do conhecimento no período 19952006. Hist Ciênc Saúde. 2009; 16(4):9991010.

2. Correa GT, Ribeiro VMB.A formação pedagógica no ensino superior e o papel da pós-graduação stricto sensu. Educ Pesqui. 2013; 39(2):319-34.

3. Haddad AE, Pierantoni CR, Ristoff D, Xavier IM, Giolo J, Silva LB. A trajetória dos cursos de graduação na área da saúde: 1991-2004. Brasília: Instituto Nacional de Estudos e Pesquisas Educacionais Anísio Teixeira; 2006. $531 \mathrm{p}$ 
4. Lei no 10.861, de 14 de abril de 2004. Institui o Sistema Nacional de Avaliação da Educação Superior - SINAES e dá outras providências. Diário Oficial da União. 15 Abr 2004.

5. Polidori MM, Marinho-Araújo CM, Barreyro GB. SINAES: perspectivas e desafios na avaliação da educação superior brasileira. Ensaio: Aval Pol Públ Educ. 2006; 14(53):425-36.

6. Lampert JB. Avaliação institucional nos cursos de graduação da área da saúde: avaliar o quê e para quê? Cadernos ABEM. 2009; 5:45-55.

7. Pessoa TRRF, Noro LRAN. Caminhos para a avaliação da formação em Odontologia: construção de modelo lógico e validação de critérios. Ciênc Saúde Coletiva. 2015; 20(7):2277-90.

8. Bardin L. Análise de Conteúdo. Lisboa: Edições 70, 2009.

9. Forte FDS, Pessoa TRRF, Freitas CHSM, Pereira CAL, Carvalho Júnior PM. Reorientação na formação de cirurgiõesdentistas: o olhar dos preceptores sobre estágios supervisionados no Sistema Único de Saúde (SUS). Interface (Botucatu). 2015; 19(suppl.1):831-43.

10. Toassi RFC, Davoglio RS, Lemos VMA. Integração ensino-serviçocomunidade: o estágio na atenção básica da graduação em Odontologia. Educ Rev. 2012; 28(4):223-42.

11. Klelba ME, Colliselli L, Dutra AT, Muller ES. Trilha interpretativa como estratégia de educação em saúde: potencial para o trabalho multiprofissional e intersetorial. Interface (Botucatu). 2016; 20(56):217-26.

12. Rego, C, Batista SH. Desenvolvimento docente nos cursos de medicina: um campo fecundo. Rev Bras Educ Med. 2012; 36(3):317-24.
13. Munguba MCS. Educação na saúde: sobreposição de saberes ou interface? Rev Bras Prom Saúde. 2010; 23(4):295-96.

14. Freitas CM, Freitas CASL, Parente JRF, Vasconcelos MIO, Lima GK, Mesquita KO, Martins SC, Mendes JDR. Uso de metodologias ativas de aprendizagem para a educação na saúde: análise da produção científica. Trab Educ Saúde. 2015; 13(suppl.2):117-30.

15. Pedrosa IL, Lira GA, Oliveira B, Silva MSML, Santos MB, Silva EA, Freire DMC. Uso de metodologias ativas na formação técnica do agente comunitário de saúde. Trab Educ Saúde. 2011; 9(2):319-32.

16. Franco LLMM, Soares EF, Martorell LB, Marcelo VC. O professor do curso de Odontologia: sua formação e os desafios frente às exigências atuais. Rev Prof Docente. 2009; 9(20):57-74.

17. Medina-Moya JL, Prado ML. El curriculum de enfermería como prototipo de tejné: racionalidad instrumental y tecnológica. Texto Contexto Enferm. 2009; 18(4):617-26.

18. Silva LHA, Ferreira FC. A importância da reflexão compartilhada no processo de evolução conceitual de professores de ciências sobre seu papel na mediação do conhecimento no contexto escolar. Ciênc Educ. (Bauru). 2013; 19(2):425-38.

19. Gatti BA. Avaliação e qualidade do desenvolvimento profissional docente. Avaliação ～(Campinas). 2014; 19(2):373-84.

20. Forte AM, Flores MA. Potenciar o desenvolvimento profissional e a colaboração docente na escola. Cad Pesqui. 2012; 42(147):900-19.

21. Chaves SE, Ceccim RB. Avaliação externa no Ensino Superior na área da saúde: inquietações e a dimensão das margens. Interface (Botucatu). 2015; 
19(55):1233-42.

22. Guerra GKS, Machado LB. Representações sociais de avaliação processual construídas por professoras. Ensaio: Aval Pol Públ Educ. [online]. 2011; 19(71):363-80.

23. Resolução CNE/CES no 3, de 20 de junho de 2014. Institui Diretrizes Curriculares Nacionais do Curso de Graduação em Medicina e dá outras providências. Diário Oficial da União. 23 Jun 2014.

\section{Correspondência para:}

Luiz Roberto Augusto Noro e-mail: luiz_noro@hotmail.com Universidade Federal do Rio Grande do Norte Avenida Salgado Filho, 1787 59056-000 Natal/RN 